\title{
Efficacy and toxicities of low-temperature plasma radiofrequency ablation for the treatment of laryngomalacia in neonates and infants: a prospective randomized controlled trial
}

\author{
Hongming Xu, Fang Chen, Yangyang Zheng, Xiaoyan Li \\ Department of Otolaryngology-Head and Neck Surgery, Children's Hospital of Shanghai, Shanghai Jiao Tong University, Shanghai, China \\ Contributions: (I) Conception and design: $\mathrm{H} \mathrm{Xu}, \mathrm{X} \mathrm{Li}$; (II) Administrative support: $\mathrm{H} \mathrm{Xu}, \mathrm{F}$ Chen; (III) Provision of study materials or patients: F \\ Chen, Y Zheng; (IV) Collection and assembly of data: H Xu, F Chen; (V) Data analysis and interpretation: F Chen, Y Zheng; (VI) Manuscript \\ writing: All authors; (VII) Final approval of manuscript: All authors. \\ Correspondence to: Prof. Xiaoyan Li. Department of Otolaryngology-Head and Neck Surgery, Children's Hospital of Shanghai, Shanghai Jiao Tong \\ University, 355th Luding Road, Shanghai 200062, China. Email: submissionent@163.com; lixy@shchildren.com.cn.
}

Background: Laryngomalacia is the most common cause of stridor in neonates and infants, and supraglottoplasty is the mainstay of surgical treatment. Although low-temperature plasma radiofrequency ablation (LTP-RFA) using coblation technology has been used for treating laryngomalacia, it is still lack of high-quality clinical evidence. Therefore, we conduct this prospective randomized study to clearly define the role of LTP-RFA for the treatment of laryngomalacia in neonates and infants.

Methods: Between Jan 2017 and Dec 2019, a total of 89 children with laryngomalacia were included for analysis. All patients were initially stratified according to the severity of laryngomalacia. Patients with severe laryngomalacia were randomly assigned to receive LTP-RFA or traditionally surgical supraglottoplasty, while patients with moderate laryngomalacia were assigned to LTP-RFA or observation. The primary end point was the efficacy and toxicities of LTP-RFA by assessing the changes of clinical score and visual analogue scale (VAS) symptom score. The total score was the combination of clinical score with VAS score.

Results: Of the 89 children, 40 children presented with severe laryngomalacia, and the remaining 49 children were diagnosed as moderate laryngomalacia. The median age was 68 days (range, 19 to 337 days). For children with severe laryngomalacia, our results showed that LTP-RFA treatment significantly reduced the operative time $(5.55 \pm 1.66$ vs. $18.7 \pm 5.31 \mathrm{~min}, \mathrm{P}<0.001)$, length of hospital stay $(6.71 \pm 1.15$ vs. $7.95 \pm 1.55$ days, $\mathrm{P}=0.008)$ and the amount of intraoperative hemorrhage $(1.71 \pm 1.79$ vs. $4.90 \pm 1.82, \mathrm{P}<0.001)$ when compared to traditionally surgical supraglottoplasty, while the treatment efficacy was comparable between LTPRFA and traditionally surgical supraglottoplasty in terms of changed total score $(\mathrm{P}=0.322)$, changed clinical score $(\mathrm{P}=0.135)$ and changed VAS symptom score $(\mathrm{P}=0.559)$. Additionally, for children with moderate laryngomalacia, LTP-RFA treatment significantly improved the symptom evaluated by total score $(\mathrm{P}<0.001)$, clinical score $(\mathrm{P}<0.001)$ and VAS symptom score $(\mathrm{P}<0.001)$ in comparison with the observation group. Postoperative pneumonia was observed in 10 patients. No surgical related death was reported.

Conclusions: The present study indicated that LTP-RFA was an effective treatment option for both severe and moderate laryngomalacia in neonates and infants with a low intraoperative complication. Long-term outcomes of LTP-RFA for laryngomalacia would be reported in further studies.

Keywords: Laryngomalacia; low-temperature plasma radiofrequency ablation (LTP-RFA); efficacy; toxicity; pediatric

Submitted Apr 06, 2020. Accepted for publication Sep 18, 2020.

doi: $10.21037 /$ atm-20-3164

View this article at: http://dx.doi.org/10.21037/atm-20-3164 


\section{Introduction}

Laryngomalacia is a common disease of infancy, affecting $45-75 \%$ of all neonates and infants who present with congenital stridor (1-3). The typical manifestation is the upper airway obstruction caused by the collapse of supraglottic structures (4). In general, symptoms, typically presents itself as inspiratory stridor, would become apparent after the first 2 weeks of life, increase towards the $6^{\text {th }}$ to $8^{\text {th }}$ month of life, and then most cases would resolve between 12 and 18 months of age with minimal or no treatment. However, approximately $10-15 \%$ of cases require surgical intervention due to a severe presentation or worsening of symptom despite conservative treatment $(5,6)$. Traditional supraglottoplasty for laryngomalacia is most commonly conducted with cold steel instruments. Although traditional surgery achieves a high success rate, serious complications including bleeding, supraglottic stenosis and aspiration can occur $(7,8)$. Low-temperature plasma radiofrequency ablation (LTP-RFA) using coblation technology is a relatively new technology that has shown promise in treating pediatric laryngeal disease, with minimally invasive (9). Since Jan 2014, our institute firstly introduced LTP-RFA for the treatment of laryngomalacia, approximately 25 patients would receive LTP-RFA treatment annually. In our previously published retrospective study of 17 patients, we found that LTP-RFA was an effective and safe treatment for severe laryngomalacia, which provided better breathing, feeding and sleeping outcomes (10). However, whether LTP-RFA is superior to traditional approaches remains unknown. In addition, treatment strategy for mild-moderate laryngomalacia remains controversial although wait-and-see policy would be recommended. In a recent publication by van der Heijden et al. found that supraglottoplasty significantly reduced the improvement of laryngomalacia than wait-andsee policy (5 vs. 29 weeks, $\mathrm{P}=0.026$ ) in all severities (11). Therefore, the primary aim of the present study is to investigate the optimal treatment strategy for the treatment of laryngomalacia by comparing the short outcomes of patients with moderate and severe laryngomalacia, who have been randomly treated with LTP-RFA, traditionally surgical supraglottoplasty or wait-and-see policy. We present the following article in accordance with the CONSORT reporting checklist (available at http://dx.doi.org/10.21037/ atm-20-3164).

\section{Methods}

\section{Study design}

This study was a single institute, randomized, open-label, active-controlled, phase II trial. A total of 120 children with laryngomalacia were enrolled. After enrollment, patients were stratified according to severity of laryngomalacia. Children with severe laryngomalacia would be randomly (1:1) treated with LTP-RFA using coblation technology or traditionally surgical supraglottoplasty. For children with moderate laryngomalacia, we would take a wait-and-see strategy to observe the symptom changes in 3 months. If the clinical symptom remained or aggravated, these children would be randomly $(1: 1)$ treated with LTP-RFA using coblation technology or observation. Computer-generated random number table was used for randomization and allocation.

\section{Ethical standards}

This study was a prospective randomized controlled trial in our institution which had been approved by ethical committee of Shanghai Children's Hospital, Shanghai Jiao Tong University. The trial had been registered in Chinese Clinical Trial Registry (http://www.chictr.org.cn/ index.aspx; registration number: ChiCTR2000029098). All procedures performed in this study involving human participants were in accordance with the ethical standards of the institutional and national research committee and with the 1964 Declaration of Helsinki and its later amendments or comparable ethical standards. Informed consent was obtained from all individual participants' parents included in the study.

\section{Patient selection}

A division of severity of laryngomalacia was made on clinical presentation and endoscopic findings (3). Criteria of severity and indicators for surgical treatment in our institution included one or more of the following items: severe stridor with tachypnea, dyspnea, or hypoxia, usually present as moderate to severe laryngeal obstruction, recurrent cyanosis, sustained failure to thrive, aspiration pneumonia, feeding difficulties with choking or suffocating episodes or endoscopy showed significant dynamic airway obstruction. The diagnosis criteria for moderate laryngomalacia in the 
Table 1 Clinical score objectively assessed by the physicians

\begin{tabular}{|c|c|c|c|c|}
\hline Variable & \multicolumn{4}{|c|}{ Score } \\
\hline Laryngeal obstruction & None & I & II & III \\
\hline Supraglottic morphology in laryngoscope & Normal & & & \\
\hline Swallowing function & Normal & & & \\
\hline Polysomnography/SPO${ }_{2}$ & Normal/93-100\% & Mild OSA/85-92\% & Moderate OSA/75-84\% & Severe $\mathrm{OSA} / \leq 74 \%$ \\
\hline
\end{tabular}

OSA, obstructive sleep apnea.

present study was child presented with one or more of the following items: cough, choking, regurgitation, and feeding difficulty. In addition, mild laryngomalacia was diagnosed with inspiratory stridor with no other symptom or radiographic findings suggesting secondary airway lesion. Transnasal flexible fiberoptic laryngoscopy (FFL) would be performed for all included patients to confirm the diagnosis of laryngomalacia.

\section{Interventions}

All patients were initially stratified according to the severity of laryngomalacia. Children with severe laryngomalacia would be randomly treated with LTP-RFA using coblation technology or traditionally surgical supraglottoplasty. For children with moderate laryngomalacia, we would take a wait-and-see strategy to observe the symptom changes in 3 months. If the clinical symptom remained or aggravated, these children would be randomly treated with LTP-RFA using coblation technology or observation. The detailed surgical technique of LTP-RFA had been described in our previously studies (10). Perioperative indexes of the three groups were observed, including operative period, bleeding volume during the operation and length of hospital stay. All patients were kept intubated for 5-7 days after surgery in the intensive care unit (ICU).

\section{Outcomes}

Prior to the present study, we had established a simple evaluation system to efficiently assess the therapeutic effect of supraglottoplasty for laryngomalacia (12). The therapeutic evaluation system consisted of clinical score and visual analogue scale (VAS) score. VAS score was used for the parents of the children to assess the subjective symptoms including stridor, reflux or feeding difficulties, aspiration, dyspnea and frequency or severity of pneumonia (each of them, ranging 0-10), and the VAS score for subjective symptom ranging from 0 to 50; Clinical score was objectively determined by the physician on the children's laryngeal obstruction, supraglottic morphology in laryngoscope, swallowing function, weight and the result of polysomnography (each of them, ranging 0-10, Table 1). The total score was the combination of clinical score with VAS score (ranging 0 to 100). The changed total score, clinical score and VAS symptom score after treatment was used to compare the therapeutic effect of supraglottoplasty.

\section{Statistical analysis}

Sample size for the present study was estimated based on our previous study (12). The mean value of total score in LTP-RFA was 23.64, and the standard deviation was 6.10; the mean value of total score in traditional surgery was 8.25 , and the standard deviation was 6.26 . The mean value of total score in observation was 14.4 , and the standard deviation was 6.01. To detect a significant difference between any groups with a power of $90 \%$ and a type I error of $5 \%$ (to detect a smallest difference of any two mean with 9 , the standard deviation was 6), the calculated each group were 27 patients. Considering a $5 \%$ dropout rate, the total sample size required were 120 patients. Simple descriptive statistics were conducted (including frequencies and percentages) to determine the baseline characteristics. Continuous variables were presented as means \pm standard errors and levels of significance was calculated by independent $t$-tests. The Mann-Whitney $\mathrm{U}$ test was used to determine differences between groups for continuous non-normally distributed variables. Statistical analysis was performed using SPSS version 20.0. Statistical significance 


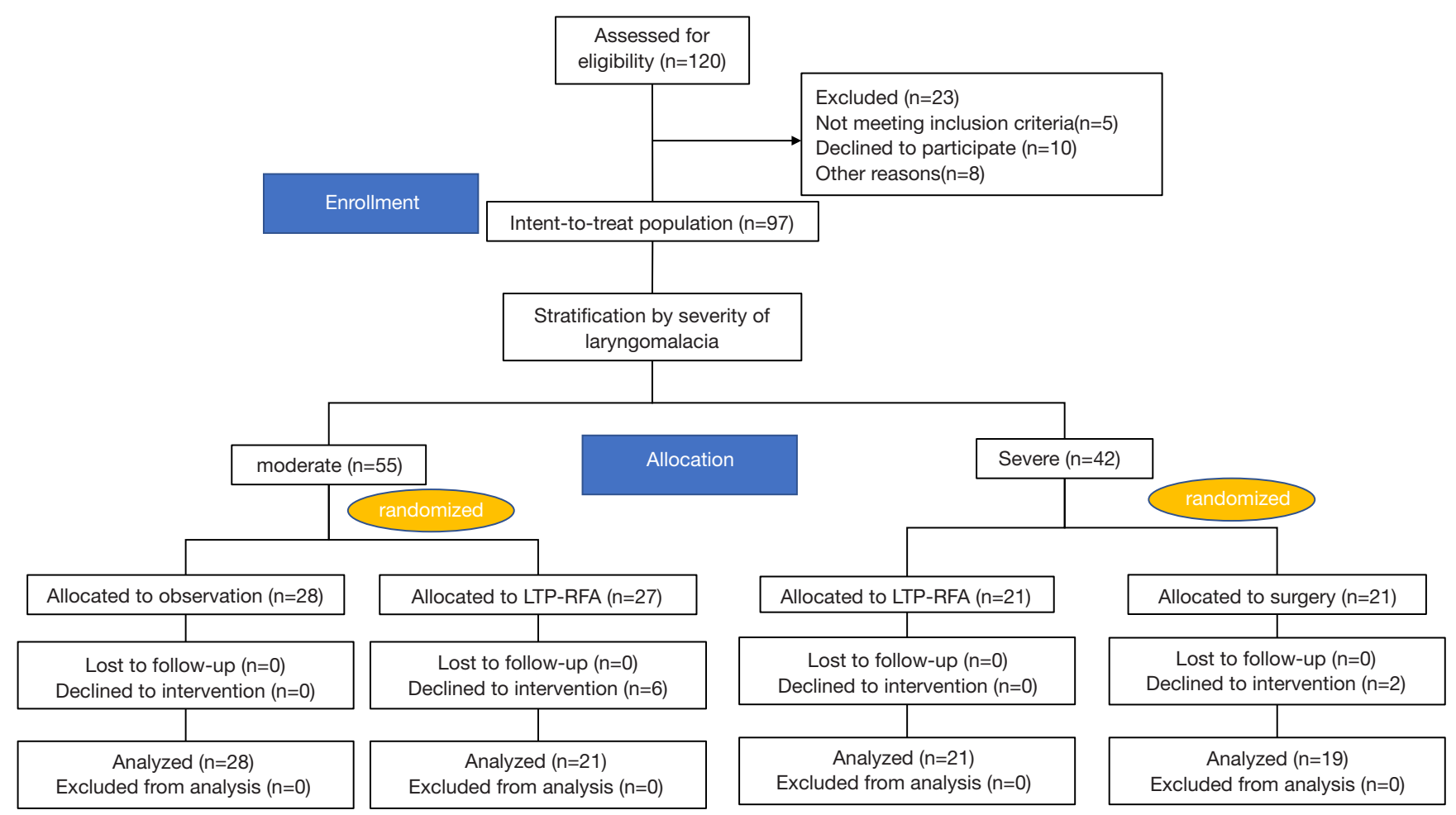

Figure 1 CONSORT diagram showing patient disposition. LTP-RFA, low-temperature plasma radiofrequency ablation.

was set at $\mathrm{P}<0.05$.

\section{Manuscript preparation}

The work has been reported in line with Consolidated Standards of Reporting Trials (CONSORT) Guidelines.

\section{Results}

\section{Study flow}

As shown in Figure 1, Between Jan 2017 and Dec 2019, a total of 120 patients were initially enrolled. Of them, 97 patients were randomized. Then, patients were stratified according to severity of laryngomalacia. A total of 42 children with severe laryngomalacia were randomized 1:1 to received LTP-RFA using coblation technology (21 children) or traditionally surgical supraglottoplasty (21 children), And 2 patients with severe laryngomalacia in the declined to intervention, thus a total of 19 children with severe laryngomalacia in the traditional surgery group were included for analysis. For 55 children with moderate laryngomalacia, 28 children were allocated to observation, and 27 children were allocated to LTP-RFA. And 6 patients with moderate laryngomalacia in the declined to intervention, thus a total of 21 children with moderate laryngomalacia treated with LTP-RFA were included for efficacy analysis.

\section{Baseline characteristics}

The median age was 68 days (range, 19 to 337 days). Fifty seven patients (64\%) were male and thirty-two patients $(36 \%)$ was female. The morphological type of laryngomalacia was seen in type II (49.4\%), type III (1.2\%) or combined types (49.4\%). Of the 89 children, 40 children presented with severe laryngomalacia, and the remaining 49 children were moderate laryngomalacia (Table 2). Comorbidity was found in 9 patients $(10.1 \%)$, the most commonly found comorbidity was lingual root cysts with 4 cases.

\section{Efficacy and safety of LTP-RFA vs. traditional surgery}

A total of 42 children with severe laryngomalacia were randomized (Figure 1). Of them, two patients in surgery 
Table 2 Baseline characteristics of included trials $(\mathrm{N}=89)$

\begin{tabular}{|c|c|}
\hline Characteristics & Value (\%) \\
\hline Age in days, median [range] & 68 [19-337] \\
\hline \multicolumn{2}{|l|}{ Sex, n (\%) } \\
\hline Male & $57(64.0)$ \\
\hline Female & $32(36.0)$ \\
\hline \multicolumn{2}{|l|}{ Severity of laryngomalacia } \\
\hline Moderate & $49(55.1)$ \\
\hline Severe & $40(44.9)$ \\
\hline \multicolumn{2}{|l|}{ Comorbidities } \\
\hline Total & $9(10.1)$ \\
\hline Neurological & $2(2.2)$ \\
\hline Lingual root cysts & $4(4.5)$ \\
\hline Pierre Robin's syndrome & $1(1.1)$ \\
\hline Spinal muscular atrophy & $1(1.1)$ \\
\hline Crouzon syndrome & $1(1.1)$ \\
\hline None & $80(89.9)$ \\
\hline \multicolumn{2}{|l|}{ Type of laryngomalacia } \\
\hline Type I & 0 \\
\hline Type II & $44(49.4)$ \\
\hline Type III & $1(1.2)$ \\
\hline Mixed type & $44(49.4)$ \\
\hline
\end{tabular}

group declined to the randomized intervention, thus 19 children finally received traditionally surgery, while 21 treated with LTP-RFA. Our results showed that LTPRFA treatment significantly decreased the operative time (5.55 \pm 1.66 vs. $18.7 \pm 5.31 \mathrm{~min}, \mathrm{P}<0.001)$ and length of hospital stay $(6.71 \pm 1.15$ vs. $7.95 \pm 1.55$ days, $\mathrm{P}=0.008)$ by using $\mathrm{T}$ test (Table 3). As for the amount of intraoperative hemorrhage was non-normally distributed variables, the Mann-Whitney U test was used. The result demonstrated that LTP-RFA treatment also significantly reduced the amount of intraoperative hemorrhage $(1.71 \pm 1.79 v s$. $4.90 \pm 1.82, \mathrm{P}<0.001$ ) when compared to traditionally surgical supraglottoplasty. As for the efficacy differences between LTP-RFA and traditionally surgical supraglottoplasty, no significant differences were found between the two groups assessed by changed total scores $(\mathrm{P}=0.322)$, changed clinical scores $(\mathrm{P}=0.135)$ and changed VAS symptom score $(\mathrm{P}=0.559$, Table 4).

\section{Efficacy of LTP-RFA vs. observation}

A total of 55 children with moderate laryngomalacia were randomized to LTP-RFA (27 children) or observation group (28 children). As six patients in the LTP-RFA declined the intervention for other reasons, 21 children in LTP-RFA and 28 in the observation groups were included for analysis (Figure 1). Our results showed that LTP-RFA treatment

Table 3 Safety comparison of LTP-RFA vs. traditional surgery

\begin{tabular}{|c|c|c|c|c|c|c|}
\hline Variable & $\mathrm{N}$ & Mean & SD & SE & $T / U^{*}$ & $\mathrm{P}$ \\
\hline Traditional surgery & 19 & 18.705 & 5.309 & 1.218 & & \\
\hline LTP-RFA & 21 & 5.5471 & 1.658 & 0.362 & & \\
\hline Length of hospital stay (day) & & & & & 158 & 0.008 \\
\hline LTP-RFA & 21 & 6.714 & 1.146 & 0.250 & & \\
\hline Postoperative hemorrhage $\left(\mathrm{cm}^{3}\right)$ & & & & & $5.559^{\star \star}$ & $<0.001$ \\
\hline Traditional surgery & 19 & 4.895 & 1.823 & 0.418 & & \\
\hline LTP-RFA & 21 & 1.714 & 1.793 & 0.391 & & \\
\hline
\end{tabular}

*, continuous normally distributed variables were calculated by independent $t$-tests. Continuous non-normally distributed variables were compared by the Mann-Whitney $U$ test. ${ }^{* *}, U$ value. $N$, number; SD, standard deviation; SE, standard error; LTP-RFA, low-temperature plasma radiofrequency ablation. 
Table 4 Efficacy comparison of LTP-RFA vs. traditional surgery

\begin{tabular}{|c|c|c|c|c|c|c|}
\hline Variable & $\mathrm{n}$ & Mean & SD & SE & $T / U^{*}$ & $P$ \\
\hline Traditional surgery & 19 & 11.211 & 7.598 & 1.743 & & \\
\hline LTP-RFA & 21 & 13.619 & 10.395 & 2.268 & & \\
\hline Changes of clinical score & & & & & $1.528^{\star *}$ & 0.135 \\
\hline LTP-RFA & 21 & 5.905 & 3.632 & 0.793 & & \\
\hline Changes of total score & & & & & 162.5 & 0.322 \\
\hline Traditional surgery & 19 & 15.474 & 9.137 & 2.096 & & \\
\hline LTP-RFA & 21 & 19.524 & 12.323 & 2.689 & & \\
\hline
\end{tabular}

*, continuous normally distributed variables were calculated by independent $t$-tests. Continuous non-normally distributed variables were compared by the Mann-Whitney $U$ test. ${ }^{* *}, U$ value. $N$, number; SD, standard deviation; SE, standard error; LTP-RFA, low-temperature plasma radiofrequency ablation; VAS, visual analogue scale.

Table 5 Efficacy comparison of LTP-RFA vs. observation

\begin{tabular}{|c|c|c|c|c|c|c|}
\hline Variable & $\mathrm{N}$ & Mean & SD & SE & $U$ & $\mathrm{P}$ \\
\hline LTP-RFA & 21 & 11.857 & 7.793 & 1.701 & & \\
\hline Observation & 28 & 2.929 & 3.126 & 0.591 & & \\
\hline Changes of clinical score & & & & & 54 & $<0.001$ \\
\hline Observation & 28 & 2.071 & 1.999 & 0.378 & & \\
\hline Changes of total score & & & & & 50 & $<0.001$ \\
\hline LTP-RFA & 21 & 19.810 & 11.587 & 2.529 & & \\
\hline Observation & 28 & 5 & 4.055 & 0.766 & & \\
\hline
\end{tabular}

$\mathrm{N}$, number; SD, standard deviation; SE, standard error; LTP-RFA, low-temperature plasma radiofrequency ablation; VAS, visual analogue scale.

significantly improved the symptom evaluated by changed total score $(\mathrm{P}<0.001)$, changed clinical score $(\mathrm{P}<0.001)$ and changed VAS symptom score $(\mathrm{P}<0.001)$ in comparison with the observation group (Table 5). The Mann-Whitney $\mathrm{U}$ test was used for the comparisons.

\section{Post-operative complications}

Post-operative pneumonia was the most common observed treatment-related complications (10 patients). For children with severe laryngomalacia, 4 cases developed pneumonia in LTP-RFA and 3 cases in traditional surgery; for children with moderate laryngomalacia, 3 cases developed pneumonia after undergoing LTP-RFA treatment, while 5 patients in observation group developed pneumonia during the follow-up time. One patient with spinal muscular atrophy in the traditional surgery group developed pneumonia after surgery, finally died as the child's parents decided to give up treatment. No surgical related deaths were reported.

\section{Discussion}

As we all known, most cases of laryngomalacia are mild and self-limited. Therefore, a majority of children would 
be treated with wait-and-see strategy, excepting for severe laryngomalacia. However, an increasing tendency towards a surgical option for mild-moderate laryngomalacia has been observed as well. In a respective case reported by Ribeiro et al. (13), the authors found that $28.2 \%$ of the children received supraglottoplasty, which was higher than previous reports $(14,15)$. In another large retrospective study conducted by van der Heijden et al. (11) demonstrated that the surgical procedure significantly shortened the time necessary to reach an improvement of symptoms in all types of laryngomalacia. As a result, the optimal treatment strategy for moderate laryngomalacia remains undetermined and there is an urgent need to clearly determine the role of supraglottoplasty for children in this setting. Additionally, although multiple retrospective studies have showed that RFA is an effective and safe treatment for severe laryngomalacia, no prospective evidence of direct comparison between the efficacy and toxicities of RFA and traditional surgery is available.

This is the first prospective four-arms randomized trial to compare the short outcomes of patients with moderate and severe laryngomalacia, who have been randomly treated with LTP-RFA, traditionally surgical supraglottoplasty or wait-and-see policy according to the severity of laryngomalacia. A total of 89 children are finally included for analysis. Among them, 40 children present with severe laryngomalacia, and the remaining 49 children are diagnosed as moderate laryngomalacia. Our results show that LTP-RFA treatment significantly reduces the operative time $(\mathrm{P}<0.001)$, length of hospital stay $(\mathrm{P}=0.008)$ and the amount of intraoperative hemorrhage $(\mathrm{P}<0.001)$ when compared to traditionally surgical supraglottoplasty, while symptom improvement based on clinical or VAS score between LTP-RFA treatment and traditional surgery are comparable. In consistent with previous reports, our results confirm that LTP-RFA treatment significantly shortens the time necessary to reach an improvement of symptoms when compared to wait-and see-strategy.

Treatment related complication is another major concern. A noteworthy finding in our study is that a total of 10 patients (14.7\%) develop post-operative pneumonia. A comparable incidence of post-operative pneumonia is observed between LTP-RFA treatment and traditional surgery. One possible reason for this finding is that all patients would be intubated for 5-7 days after surgery in the ICU in order to achieve a better epiglottal position and to avoid reconglutination of the aryepiglottic folds, and most of these post-operative pneumonia occur during this time, thus surgeons should pay more attention to the risk of developing pneumonia after surgery. Additionally, 5 patients $(17.8 \%)$ with moderate laryngomalacia pneumonia develops pneumonia during the wait-and-see time. It has been reported that comorbidities decrease the success rate of laryngomalacia, and even failures and complications of surgery are attributed to comorbidities. Comorbidity is found in $10 \%$ of our patients, which is lower than the incidence of literature comorbidity (25-47\%) (16). In our series, one patient with spinal muscular atrophy in the traditional surgery group develops pneumonia after surgery, this patient finally dies as the child's parents decide to give up treatment.

Several limitations are needed to be concerned. First of all, our population of children with laryngomalacia is selected according to inclusion criteria, which is not entirely representative of the general population of children with laryngomalacia. However, this is the first study gives an insight into treatment strategy of moderate laryngomalacia. Secondly, the follow-up time of the present study is relative short, thus long-term outcomes of children treated LTPRFA would be reported in our further study. Thirdly, the quality of life (QoL) of children with laryngomalacia is not assessed in the present study, although previous study shows that an improvement on the QoL could be reached with appropriate treatment (17).

\section{Conclusions}

This is the first largest prospective randomized controlled trial to investigate the efficacy and toxicities of LTP-RFA versus traditional surgery or observation for the treatment of laryngomalacia. The present study indicates that LTPRFA is an effective and safe treatment option for both severe and moderate laryngomalacia in neonates and infants with a low intraoperative complication. No surgical related death is reported. Long-term outcomes of children treated with LTP-RFA for laryngomalacia would be reported in further studies.

\section{Acknowledgments}

Funding: The present work was supported by medical engineering cross project of Shanghai Jiaotong University (grant: 20194Y0165) and Clinical Research Plan of Shanghai Shenkang Hospital Development Center (grant 20873999). 


\section{Footnote}

Reporting Checklist: The authors have completed the CONSORT reporting checklist. Available at http://dx.doi. org/10.21037/atm-20-3164

Data Sharing Statement: Available at http://dx.doi. org/10.21037/atm-20-3164

Conflicts of Interest: All authors have completed the ICMJE uniform disclosure form (available at http://dx.doi. org/10.21037/atm-20-3164). The authors have no conflicts of interest to declare.

Ethical Statement: The authors are accountable for all aspects of the work in ensuring that questions related to the accuracy or integrity of any part of the work are appropriately investigated and resolved. The study was conducted in accordance with the Declaration of Helsinki (as revised in 2013). This study was a prospective randomized controlled trial in our institution which had been approved by ethical committee of Shanghai Children's Hospital, Shanghai Jiao Tong University (registration number: ChiCTR2000029098). Informed consent was obtained from all participants included in the study.

Open Access Statement: This is an Open Access article distributed in accordance with the Creative Commons Attribution-NonCommercial-NoDerivs 4.0 International License (CC BY-NC-ND 4.0), which permits the noncommercial replication and distribution of the article with the strict proviso that no changes or edits are made and the original work is properly cited (including links to both the formal publication through the relevant DOI and the license). See: https://creativecommons.org/licenses/by-nc-nd/4.0/.

\section{References}

1. Zoumalan R, Maddalozzo J, Holinger LD. Etiology of stridor in infants. Ann Otol Rhinol Laryngol 2007;116:329-34.

2. Thorne MC, Garetz SL. Laryngomalacia: Review and Summary of Current Clinical Practice in 2015. Paediatr Respir Rev 2016;17:3-8.

3. Landry AM, Thompson DM. Laryngomalacia: disease presentation, spectrum, and management. Int J Pediatr 2012;2012:753526.

4. Kay DJ, Goldsmith AJ. Laryngomalacia: a classification system and surgical treatment strategy. Ear Nose Throat J
2006;85:328-31, 36.

5. Dobbie AM, White DR. Laryngomalacia. Pediatr Clin North Am 2013;60:893-902.

6. McCaffer C, Blackmore K, Flood LM. Laryngomalacia: is there an evidence base for management? J Laryngol Otol 2017;131:946-54.

7. Richter GT, Thompson DM. The surgical management of laryngomalacia. Otolaryngol Clin North Am 2008;41:83764, vii.

8. Thompson DM. Laryngomalacia: factors that influence disease severity and outcomes of management. Curr Opin Otolaryngol Head Neck Surg 2010;18:564-70.

9. Ching HH, Spinner AG, Reeve NH, et al. A novel technique for unilateral supraglottoplasty. Int J Pediatr Otorhinolaryngol 2018;104:150-4.

10. $\mathrm{Pu} \mathrm{S,} \mathrm{Xu} \mathrm{H,} \mathrm{Li} \mathrm{X.} \mathrm{Supraglottoplasty} \mathrm{in} \mathrm{neonates} \mathrm{and}$ infants: A radiofrequency ablation approach. Medicine (Baltimore) 2018;97:e9850.

11. van der Heijden M, Dikkers FG, Halmos GB. Treatment outcome of supraglottoplasty vs. wait-and-see policy in patients with laryngomalacia. Eur Arch Otorhinolaryngol 2016;273:1507-13.

12. Pu SL, Li XY. [Therapeutic evaluation of modified supraglottoplasty for laryngomalacia]. Lin Chung Er Bi Yan Hou Tou Jing Wai Ke Za Zhi 2019;33:1072-5; 80.

13. Ribeiro J, Julio S, Dias C, et al. Supraglottoplasty in children with laryngomalacia: A review and parents' appraisal. Am J Otolaryngol 2018;39:613-7.

14. Preciado D, Zalzal G. A systematic review of supraglottoplasty outcomes. Arch Otolaryngol Head Neck Surg 2012;138:718-21.

15. Ayari S, Aubertin G, Girschig H, et al. Management of laryngomalacia. Eur Ann Otorhinolaryngol Head Neck Dis 2013;130:15-21.

16. Hoff SR, Schroeder JW Jr, Rastatter JC, et al. Supraglottoplasty outcomes in relation to age and comorbid conditions. Int J Pediatr Otorhinolaryngol 2010;74:245-9.

17. Kilpatrick LA, Boyette JR, Hartzell LD, et al. Prospective quality of life assessment in congenital laryngomalacia. Int J Pediatr Otorhinolaryngol 2014;78:583-7.

Cite this article as: $\mathrm{Xu} \mathrm{H}$, Chen F, Zheng Y, Li X. Efficacy and toxicities of low-temperature plasma radiofrequency ablation for the treatment of laryngomalacia in neonates and infants: a prospective randomized controlled trial. Ann Transl Med 2020;8(21):1366. doi: 10.21037/atm-20-3164 